\title{
Multiple semantic encoding of homophones and homographs in contexts biasing dominant or subordinate meanings
}

\author{
ROBERT E. WARREN, NATALIE TERBOVIC WARREN, JOHN P. GREEN, \\ and JAN H. BRESNICK \\ Columbia University, New York, New York 10027
}

\begin{abstract}
The resolution of lexical ambiguity was studied in two experiments. While subsequent selection would appear to limit longer term storage to one meaning of an ambiguous word, multiple initial encoding of homophones and homographs was indicated by the intrusion of their alternative meanings across trials in a Brown-Peterson paradigm. In Experiment 1 subjects heard four words and then engaged in a 10-sec distractor task. Written recall was then demanded. On context trials a homophone with two distinct alternative meanings was presented with other words biasing either a dominant or subordinate meaning, for example, look, stare, peer, glance. On the next trial words related to an alternative meaning were presented, for example, dock, wharf, quay, jetty. Intrusions of the graphic forms of alternative meanings (e.g., PIER) into critical trial recall occurred whether the dominant or subordinate meaning of the homophone had been biased by context. Experiment 2 employed visual presentation of homographs, for example, GOLD, IRON, LEAD, and oral recall, but was logically similar to Experiment 1. Intrusions of the alternative codings of homographs across trials again occurred, for example, GUIDE, DIRECT, lead, regardless of the meaning originally biased by context.
\end{abstract}

The resolution of lexical ambiguity has proved to be one of the more refractory problems in the study of language comprehension. While it is generally recognized that most words have more than one distinct meaning, individuals are usually aware of only one meaning in any given utterance. The problem, then, is how one resolves this basic ambiguity and selects one meaning from those available. In most instances, of course, a word is not encountered in isolation but rather embedded in a context that is related to one of its possible meanings. Presumably, it is context that somehow permits the selection of one meaning from the available alternatives. It remains to be shown, however, at what point in the encoding process this selection takes place and what the fate of unselected meanings might be.

Three general views of the encoding process for ambiguous words have been advanced. Their differences lie in the number of meanings an ambiguous word is thought to activate, the mechanism and timing of selection, and the effects of the relative frequency of a word's possible meanings. First, what might be called unitary encoding models (e.g., Schvaneveldt, Meyer, \&

Requests for reprints should be sent to Robert E. Warren, Bell Laboratories, 6200 East Broad Street, Columbus, Ohio 43213. Some of the data from Experiment 1 were reported at the meeting of the Psychonomic Society, St. Louis, Missouri, November 1976. The authors wish to express their appreciation for access to the facilities of the Center for Cognitive Studies, University of Oregon, during testing for Experiment 1.
Becker, 1976) assert that only one meaning of a word is ever active during encoding and that this meaning is completely determined by the context in which the word occurs. Second, multiple encoding models (e.g., Conrad, 1974; Foss \& Jenkins, 1973; Warren \& Warren, 1976) suggest that at some early stage of encoding all meanings of a word are active, and only at some later point is a selection based on context made. Finally, ordered activation models (Hogaboam \& Perfetti, 1975) assert that when an ambiguous word is encountered its meanings are activated in order of their relative frequency of usage (dominance) until a meaning that matches context is located. The most frequent (dominant) meaning is examined first and if a match is made no further meanings become active. If, on the other hand, a less frequent (subordinate) meaning is the one to match context, it and all meanings more frequent than it will have been activated.

When evaluating the various encoding hypotheses, one must distinguish three possible facets of the encod. ing process: the activation of meaning representations, the processes which lead to a selection of one meaning over others, and the storage or other subsequent use of such selected representations. Evidence that only a single meaning of a word is available some time after processing is complete does not preclude the possibility that multiple semantic representations of the word were active and available earlier. Work on the recognition of nouns presented in context by Light and CarterSobell (1970), for example, shows that only a single 
representation of a word can be retrieved 6-7 min after presentation. These investigators presented words such as JAM in one context (e.g., strawberry JAM) and tested their recognition in other contexts (e.g., traffic JAM). The presence of only one meaning representation was indicated by reduced performance under these conditions compared to those in which recognition context biased the same meaning (e.g., grape JAM). This, however, can indicate only the end state of the encoding process and does not necessarily imply an equally restrictive representation of alternative meanings during initial encoding.

Similarly, evidence that the level of general processing capacity available for other tasks is affected by the presence or absence of ambiguity may only reflect changes in those portions of the encoding process that require such capacity. It might be the case, for example, that the activation of the meaning representations of words in memory does not require capacity, whereas the selection of one meaning from a number which are activated simultaneously does take capacity. Studies by Foss and his colleagues (Foss, 1970; Foss \& Jenkins, 1973) have used a phoneme monitoring task as a measure of the processing demands of lexical items embedded in sentences. Subjects in the experiments listened to sentences in which a target phoneme followed either an ambiguous or unambiguous word. Reaction time to detect the phoneme was longer following ambig. uous words, indicating that such words increased local processing demands. Foss argued that the increased processing demands of ambiguous words were a byproduct of the activation of their multiple meaning representations, and demonstrated that such effects were not changed by the introduction of contextual material that resolved the lexical ambiguity. Subsequent research using the same paradigm, however, has shown that sufficiently strong contexts eliminate the ambiguity effect (Swinney \& Hakes, 1976). In any case, these results may only reflect changes in the ease with which selection proceeds and leave open the question of whether single or multiple meanings are activated initially.

In an attempt to study the very early stages of the encoding of ambiguous words, Conrad (1974) presented subjects with sentences in which the final word was a homonym. Words preceding the homonym were either neutral with respect to its various possible meanings or related to one particular meaning, for example, "The sailors enjoyed the port" or "The sailors drank the port." Immediately following the last word in the sentence, subjects were required to name the color of the ink in which a visual test word, such as WINE or HARBOR, was printed. Warren $(1972,1974)$ has shown that the latency of color naming in this situation increases if subjects have encountered material semantically related to the word serving as the carrier for the color just prior to its presentation, relative to latencies observed when it is tested after unrelated material. Conrad (1974) found that both WINE and HARBOR produced such delays in color naming when tested after either the ambiguous or unambiguous presentation of PORT. Since associates of both meanings of PORT appear to have been affected equally, regardless of contextual information, Conrad suggested that, at least initially, both meanings of a homonym are available during its encoding.

On the other hand, Schvaneveldt, Meyer, and Becker (1976) found no indication that multiple meanings of homonyms were activated during encoding. These investigators presented their subjects with series of three successive letter strings. For each of the strings the subject was required to decide whether or not it was a word. On some trials a homonym appeared as the second word and was preceded by a word that biased one interpretation (e.g., RIVER, BANK). The third word was chosen to be related to the alternative meaning of the homonym (e.g., MONEY). Other work on the lexical decision task (Meyer, Schvaneveidt, \& Ruddy, Note 1) has shown that consecutive presentation of semantically related words produces faster lexical decisions. If all meanings of BANK are activated regardless of context, then one would expect that the subjects' decisions regarding the lexicality of MONEY would be facilitated. Contrary to this expectation, no facilitation was observed.

While producing conflicting results with regard to the unitary and multiple encoding models, none of the experiments cited has provided a direct test of the ordered activation model. It is possible that evidence in favor of either the multiple or unitary encoding model might be the result of the unintentional choice of experimental contexts that consistently bias either the subordinate or dominant meanings of the ambiguous words tested. That is, if the ordered activation hypothesis is true, and the dominant meanings of words were consistently biased, then one would obtain results that would appear to support the unitary encoding model. Only one meaning would ever become active because the most common meaning would match context and no others would be activated. On the other hand, if the subordinate meanings of words were consistently biased, one would obtain results supporting the multiple encoding model. Since context would not match the dominant meaning activated first, subordinate meanings would become active as well.

The present experiments utilized a method developed by Warren and Warren (1976) which exploits the multiple physical representations of homophones and homographs to study their multiple meaning representations. Although they are relatively rare in English, homophones and homographs provide extreme cases against which more general hypotheses may be tested. If a graphic or phonemic physical form that actually represents two semantically distinct words could be shown to activate 
both regardless of context, for example, then the multiple encoding hypothesis would receive support not only for the tested case but also with regard to those words that are polysemous. That is, if two completely independent semantic representations were activated by the same stimulus, it might be reasonable to believe that multiple, highly similar semantic representations would be activated in the same fashion.

Warren and Warren (1976) presented subjects with semantically homogeneous word triads in a modified Brown-Peterson paradigm. On what they termed "context" trials, subjects heard (saw) a list of three words containing a homophone (homograph) and other words related to one of its meanings, for example, HELPLESS, weak, FRAGILE. Subjects then engaged in a distractor task prior to written (oral) recall. On the immediately subsequent critical trial, words related to an alternative meaning of the homophone (homograph) were presented, for example, YEAR, MONTH, DECADE. Warren and Warren argued that, if unitary encoding models are correct, only one meaning of an ambiguous word should be active during the encoding of the context-trial material and this meaning should never appear as an intrusion in the recall of the critical-trial words. That is, subjects should never recall YEAR, MONTH, weak. As in the release-from-proactive-inhibition paradigm (Wickens, 1970), a distinct semantic representation of a word should provide subjects sufficient cues to allow its exclusion from the recall of a group of words semantically unrelated to it. On the other hand, if multiple encoding models are correct, then one might expect both weak and WEEK to be active during contexttrial encoding and the occasional intrusion of the latter into critical-trial recall. That is, subjects might recall YEAR, MONTH, WEEK. For both homophones and homographs, such intrusions occurred often enough to justify the conclusion that multiple meanings had been active.

In the present studies the same paradigm is employed but the form of the ambiguous word biased by context is systematically varied. This should allow a direct test of the ordered activation model of encoding as well as of the unitary and multiple encoding models. The three models would appear to make rather clear predictions regarding the occurrence of intrusions in this experimental paradigm. In the case of a context that biases the dominant meaning of an ambiguous word, the unitary encoding model and the ordered activation model predict that only one meaning of the word will be activated, hence no intrusions should occur. In the case of a context that biases a subordinate meaning of an ambiguous word, the unitary model again predicts no activation of alternative meanings and no intrusions. The ordered activation model, however, predicts that the dominant meaning of the word will be activated as well as the subordinate meaning, so intrusions of the dominant meaning may occur. The multiple encoding hypoth- esis predicts that both meanings of an ambiguous word will be activated, regardless of context, and intrusions will occur no matter which meaning is biased. Experiment 1 employed homophones embedded in a threeword context to test these predictions.

\section{EXPERIMENT 1}

\section{Method}

Subjects. Forty male and 40 female subjects from the Cognitive Studies Center, University of Oregon, were tested in small groups. They were paid $\$ 2$ for their participation.

Design and Procedure. Subjects listened to the prerecorded stimulus material over a loudspeaker. Each trial began with the word READY followed by four test words presented at a rate of one per second. Immediately after the last test word, subjects engaged in a rehearsal preventing task for $10 \mathrm{sec}$ and then heard the word RECALL. Fifteen seconds were allowed for recall, and subjects were encouraged to write down four words for each trial, even if they were unsure of a particular word.

Recall of the test words and the results of the distractor task were written on individual-trial response slips arranged in booklet form. For use in the distractor task, every slip had a series of 24 letters, each followed by a blank. During the retention interval, subjects were to proceed through the series, writing down in the blank next to each letter the letter preceding it in the alphabet by two places. For example, if the letter $C$ was encountered, the letter $A$ was the appropriate response. Letters were ordered using the random alphabets prepared by Hintzman (1966), excluding the letters A and B. Test word recall was recorded in a separate blank. Once subjects had completed recall for a trial they turned to the next response slip in the booklet and were not permitted to change or to refer back to any previous trial's recall.

Eight stimulus tapes were prepared and each tape was used for 10 subjects. All tapes consisted of 2 practice trials and 30 experimental trials. The experimental trials were made up of 15 pairs of trials, the first trial in each pair being a context trial and the second a critical trial. The eight tapes represented two versions of the four conditions formed by the combination of two dichotomous classifications: (1) whether or not the context trial contained a homophone (test vs. control condition) and (2) whether the words on the context trial were related to the dominant or the subordinate meaning of a homophone. The two versions differed in that each employed a different randomly chosen order of the 15 experimental trial pairs.

Half of the subjects were tested in the test condition and half in the control condition. Within each trial pair, subjects in the test condition received on the context trial a group of four words containing a homophone in the third position, for example, LOOK, STARE, peer, GLANCE. One of the possible interpretations of the homphone (e.g., peer) was related to the other words given on that context trial, and an alternative meaning was related to words presented on the immediately subsequent critical trial. For instance, the critical trial for the example just given was DOCK, WHARF, QUAY, JETTY, all related to the meaning of the alternative graphic form, PIER. Subjects in the control condition received the same material, except that on the context trial another word was substituted for the homophone (e.g., GAZE for peer).

For half the subjects in each condition the context-trial material was related to the dominant meaning of homophone and the critical-trial material to the subordinate meaning of that homophone. The other half of the subjects received the same material but in the opposite order, so that this group was given material related to the subordinate meaning on context trials and to the dominant meaning on critical trials. A complete example of this is shown in Table 1. 
Table 1

Examples of Critical and Control Trials

\begin{tabular}{|c|c|c|c|c|}
\hline Meaning Context & Condition & Context Trial & Critical Trial & Intrusion \\
\hline \multicolumn{5}{|c|}{ Experiment 1: Homophones } \\
\hline Dominant & $\begin{array}{l}\text { Test } \\
\text { Control }\end{array}$ & $\begin{array}{l}\text { LOOK STARE peer GLANCE } \\
\text { LOOK STARE GAZE GLANCE }\end{array}$ & $\begin{array}{l}\text { DOCK WHARF QUAY JETTY } \\
\text { DOCK WHARF QUAY JETTY }\end{array}$ & PIER \\
\hline Subordinate & $\begin{array}{l}\text { Test } \\
\text { Control }\end{array}$ & $\begin{array}{l}\text { DOCK WHARF PIER JETTY } \\
\text { DOCK WHARF QUAY JETTY }\end{array}$ & $\begin{array}{l}\text { LOOK STARE GAZE GLANCE } \\
\text { LOOK STARE GAZE GLANCE }\end{array}$ & peer \\
\hline \multicolumn{5}{|c|}{ Experiment 2: Homographs } \\
\hline Dominant & $\begin{array}{l}\text { Test } \\
\text { Control }\end{array}$ & $\begin{array}{l}\text { GUIDE DIRECT lead } \\
\text { GUIDE DIRECT STEER }\end{array}$ & $\begin{array}{l}\text { GOLD IRON COPPER } \\
\text { GOLD IRON COPPER }\end{array}$ & $L E A D$ \\
\hline Subordinate & $\begin{array}{l}\text { Test } \\
\text { Control }\end{array}$ & $\begin{array}{l}\text { GOLD IRON } L E A D \\
\text { GOLD IRON COPPER }\end{array}$ & $\begin{array}{l}\text { GUIDE DIRECT STEER } \\
\text { GUIDE DIRECT STEER }\end{array}$ & lead \\
\hline
\end{tabular}

Homophones and their meaning dominance levels were selected using Galbraith and Taschman's norms (1969). Homophones used showed consistent meaning dominance patterns across all four of the measures employed by Galbraith and Taschman. Subjects hearing the homophones produced written copies corresponding to the graphic form of the dominant meaning $77 \%$ of the time. Eighty-two percent of the free association responses to auditory presentations of the homophones were related to their dominant meanings. Average latency for finding an alternative graphic form of a homophone was $2.90 \mathrm{sec}$ for finding that form related to the dominant meaning given the graphic form matching the subordinate meaning, and $6.23 \mathrm{sec}$ for finding that form related to the subordinate meaning given the graphic form matching the dominant meaning. Average frequency in English (Kučera \& Francis, 1967) was 499.13 per million for the graphic forms of dominant meanings and 9.73 for the graphic forms of subordinate meanings. Other words presented on context and critical trials were generated by the experimenters and were chosen to match one or the other meaning of the appropriate homopnone.

\section{Results}

In order for differences in the number of intrusions to be meaningful, other aspects of the data must be considered. First, is the material on context trials sufficient to bias one interpretation of the homophone over the other? This appears to be the case since, in those instances when any graphic form of the homophone was recalled by the test groups, the graphic form whose meaning matched context was recalled $96.5 \%$ of the time for dominant contexts and $89.2 \%$ of the time for subordinate contexts.

Second, are general recall ability and item difficulty equivalent for the test and control groups? To resolve this question, overall error scores for critical-trial recall were determined for each subject, where an error was defined as failure to recall an item on the presented list. An analysis of variance was performed on these scores, with context type (dominant, subordinate) and condition (test, control) as factors. No difference between test and control groups was evident $(F<1)$. Context type, however, proved significant $[F(1,76)=$ $7.56, \mathrm{p}<.01]$. Subjects who received the material related to the subordinate meaning of the homophone on critical trials made more errors than those who received material related to the dominant meaning.
The interaction between the two factors was not significant $(F<1)$. This pattern of results suggests that material related to the subordinate meaning was more difficult to remember. If intrusion frequency follows recall failure frequency, one would expect a greater number of homophone intrusions in both the test and control groups that heard material related to subordinate meanings on critical trials. As shall be demonstrated, however, the opposite pattern of results was obtained.

In order to evaluate the various encoding hypotheses, one must know whether there are more intrusions of the alternative graphic form of a homophone into the critical-trial recall of the test group subjects and whether this difference is consistent across the dominant and subordinate context conditions. Recall on critical trials was examined for the intrusion of words matching the alternative graphic form of the homophone presented to the test groups on the just-prior context trial. The appearance of PIER in the recall of DOCK, WHARF, QUAY, etc., for example, was scored as a homophone intrusion. In scoring the protocols of the test groups, an intrusion on a critical trial was counted only if the subject had previously included the other graphic form of the homophone in recall of the context-trial material. If the intrusion of PIER, for example, had been preceded by recall of LOOK, STARE, PIER, GLANCE instead of peer, the intrusion would not have been counted. Similarly, such an intrusion was not counted if the subject failed to recall any graphic form of the homophone. In a parallel fashion, an intrusion found in a control group protocol was not counted if it had been preceded in the recall of the prior context trial by the production of a graphic form matching the homophone heard by the test groups. For example, if the intrusion of PIER by a control subject had been preceded by recall of LOOK, STARE, peer, GLANCE, even though the individual had never heard peer, the intrusion would not have been counted.

Intrusion frequencies in the four groups are shown in Figure 1. An analysis of variance was performed on these data, with context type (dominant, subordinate) and condition (test, control) as factors. A $\sqrt{x+.5}$ trans- 


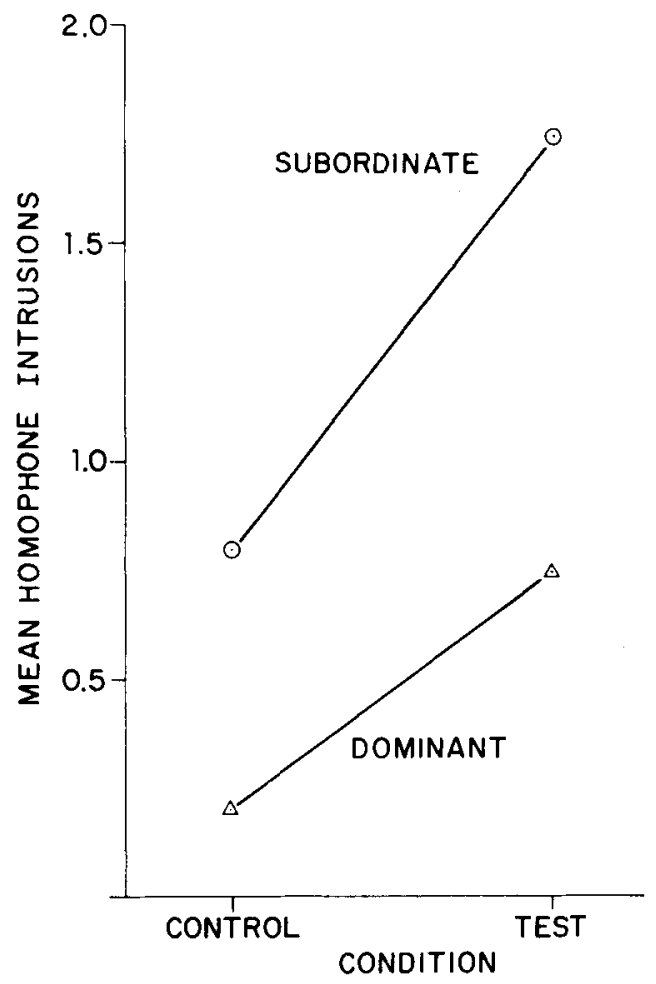

Figure 1. Homophone intrusions on critical trials for groups hearing material related to the subordinate or dominant form on context trials.

form, recommended by Myers (1966) for this type of frequency count data, was employed. More intrusions were produced by test groups than by control groups $[F(1,76)=8.23, p<.005]$. More intrusions also occurred when the context-trial material was related to the subordinate meaning of the homophone than when it was related to the dominant meaning $[F(1,76)=12.71, p<.001]$. There was no significant interaction of these factors $(F<1)$. As an estimate of the generality of these results beyond this particular sample of homophones, a second analysis was performed using homophones as the unit of analysis and collapsing across subjects. The same pattern of results was obtained [condition, $\mathrm{F}(1,14)=30.39, \mathrm{p}<.001$; context type, $F(1,14)=6.59, p<.025$; interaction, $F(1,14)=1.02]$.

To check the possibility that test group subjects simply tended to produce more intrusions of any type, homophone intrusion frequency was examined as a proportion of the total instrusions made by each group on critical trials. The proportions for test and control groups were $16.2 \%$ and $2.8 \%$ for subjects who had received material related to the dominant form of the homophone on context trials and $38.1 \%$ and $17.4 \%$ for those receiving material related to the subordinate form on context trials. Actual frequencies of homophone intrusions, other intrusions, and omissions are shown in Table 2.

\section{Discussion}

It appears that none of the three encoding models provides a completely satisfactory account of the data from Experiment 1. The most conspicuous failure is the unitary encoding model. Contrary to its prediction, intrusions occur in both the dominant and subordinate context conditions. The ordered activation model is only slightly more successful. The occurrence of intrusions in the dominant context condition cannot be accounted for by this model, since only one representation of a homophone should have been activated in this situation. This model does, however, make what appears to be a useful distinction between the dominant and subordinate meanings of a homophone. Dominant meanings appear to be more available in general than subordinate meanings, hence their higher intrusion rate in both test and control conditions. While it is possible that this difference in availability is due to the inadvertent generation of critical-trial contexts that elicit dominant meanings more often, it more likely reflects some basic characteristic of the memory system for words. Multiple encoding models, while successful in predicting the pattern of intrusions in the test and control conditions, must incorporate some mechanism to account for the difference in availability of dominant and subordinate meanings. A possible solution to this problem is presented in the discussion following Experiment 2, which served to extend the findings of Experiment 1 to the case of homographs embedded in a two-word context.

\section{EXPERIMENT 2}

\section{Method}

Subjects. Fifty-two male and 12 female undergraduates at Columbia University served as subjects as part of a course requirement.

Design and Procedure. Subjects were tested individually in 1/2-h sessions. Experimental stimuli were presented via a Gerbrands projection tachistoscope on a rear-projection screen, and initial instructions and individual-trial timing signals were prerecorded and presented auditorily. Each trial began with the prerecorded word READY, followed by a 3-sec exposure of three words arranged in a column. This triad in turn was replaced by a threedigit number from which the subject was to count backward by threes until the word RECALL was heard. Counting was

Table 2

Critical-Trial Error Frequency by Type of Error and Condition: Experiment 1

\begin{tabular}{clrcc}
\hline \multirow{2}{*}{$\begin{array}{c}\text { Meaning } \\
\text { Context }\end{array}$} & Condition & $\begin{array}{l}\text { Homo- } \\
\text { phone }\end{array}$ & Other & Omissions \\
\hline \multirow{2}{*}{ Dominant } & Test & 21 & 109 & 110 \\
& Control & 4 & 138 & 115 \\
Subordinate & Test & 43 & 70 & 69 \\
& Control & 16 & 76 & 67 \\
\hline
\end{tabular}


paced by a metronome beating at 45 clicks/min, and subjects were told to count back one step with each click. Prior to the experiment proper, subjects were given practice in backward counting at that pace. The RECALL signal followed trial onset by 15 sec. At this point, subjects were to recall aloud as much of the word triad as possible and were encouraged to produce three words even if they were unsure of any particular item. Fifteen seconds were allowed for recall. Ten seconds into the recall period the prompt ANY GUESSES? was heard and served as a reminder to subjects to recall three words on each trial. The interval between trial onsets was $30 \mathrm{sec}$. Recall was taperecorded during the experimental session and transcribed later.

Eight stimulus sets were prepared and each set was used with eight subjects. All sets consisted of 2 practice trials and 30 experimental trials. The experimental trials were composed of 15 pairs of trials, the first trial in each pair a context trial and the second a critical trial. The eight sets represented two versions of the four conditions formed by the combination of two dichotomous variables: (1) whether or not the context trial contained a homograph (test and control condition, respectively) and (2) whether the words on the context trial were related to the dominant or subordinate meaning of a homograph. The two versions differed in that each employed a different randomly chosen order of the 15 experimental trial pairs.

Half of the subjects were tested in the test condition and half in the control condition. On the context trial within each trial pair, the test group received a word triad containing as its last member a homograph, for example, GOLD, IRON, LEAD. Homographs always appeared as the bottom word in the triad column. The meaning of one of the possible phonemic forms of the homograph (e.g., $L E A D$ ) was related to the other words on the context trial, whereas the meaning of an alternative phonemic form was related to the words presented on the immediately subsequent critical trial. For instance, the critical trial for the example just given was GUIDE, DIRECT, STEER, all of which are related to the alternative phonemic form lead. Subjects in the control groups received the same material, except that another word was substituted for the homograph on the context trial (e.g., COPPER for $L E A D$ ).

For half the subjects the context-trial material was related to the dominant meaning of the homograph and the critical-trial material to the subordinate meaning. The other half of the subjects received the same material but in the opposite order, so that context trials biased the subordinate meaning of the homograph. An example of this arrangement is shown in Table 1. Homographs were selected and their meaning dominance levels determined from the definitional frequency norms presented by Warren, Bresnick, and Green (1977). The average frequency of definitions given to the graphic representation of a homograph was .691 for the dominant meanings and .230 for subordinate meanings. Context words were generated by the experimenters.

\section{Results}

The same three questions posed with regard to homophone data are relevant here. Once again the context material appeared sufficient to bias the appropriate interpretation of the homographs. Examination of homograph recall in test groups showed that, in those cases where either phonemic form was recalled, the form matching context was produced $98.6 \%$ of the time for dominant contexts and $82.7 \%$ of the time for subordinate contexts.

An analysis of variance on critical-trial error scores parallel to that done for homophones was performed to test the equivalence of general recall ability and item difficulty in the four groups of subjects. None of the

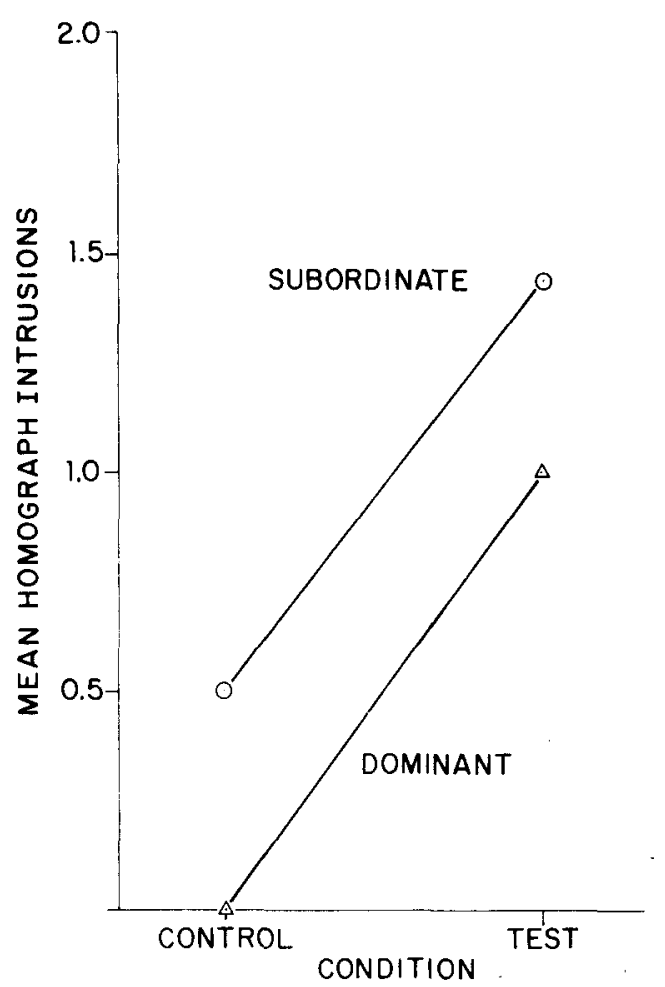

Figure 2. Homograph intrusions on critical trials for groups seing material related to the subordinate or dominant form on context trials.

factors in the analysis was significant. It appears, then, that any fluctuation in homograph intrusion frequency on critical trials cannot be attributed to differences in general recall ability or item difficulty.

Homograph intrusions on critical trials were scored using rules directly comparable to those developed in Experiment 1. Homograph intrusion frequency for the four groups is shown in Figure 2. An analysis of variance was performed on these data, with context type (dominant, subordinate) and condition (test, control) as factors. A $\sqrt{\mathbf{x}+.5}$ transform was used. The test groups produced more homograph intrusions than the control groups $[F(1,60)=24.13, p<.001]$. Subjects who received context-trial material related to the subordinate meanings of homographs produced more intrusions than those in the dominant context groups $[F(1,60)=6.97$, $\mathrm{p}<.025]$. The Context by Condiiton interaction was not significant $(F<1)$. As an estimate of the generality of these results to other samples of homographs, a second analysis was done using homographs as the unit of analysis, collapsing across subjects. A similar pattern of results emerged [condition, $\mathrm{F}(1,14)=20.15, \mathrm{p}<.001$; context type, $\mathrm{F}(1,14)=6.38, \mathrm{p}<.025$; interaction, $\mathrm{F}<1]$.

As a check on the possibility that test groups tended to produce more intrusions of any type (and hence more homograph intrusions), homograph intrusion frequency on critical trials was examined as a proportion of the total intrusions made by each group. For subjects in the subordinate context groups these proportions were 
Table 3

Critical-Trial Error Frequency by Type of Error and Condition: Experiment 2

\begin{tabular}{clrrc} 
& & \multicolumn{2}{c}{ Intrusions } \\
\cline { 3 - 4 } Meaning & Condition & $\begin{array}{c}\text { Homo- } \\
\text { phone }\end{array}$ & Other & Omissions \\
\hline \multirow{2}{*}{ Context } & Test & 16 & 46 & 61 \\
\multirow{3}{*}{ Subordinate } & Control & 0 & 44 & 66 \\
& Test & 26 & 53 & 34 \\
& Control & 8 & 49 & 70 \\
\hline
\end{tabular}

$32.9 \%$ and $14.0 \%$ in the test and control conditions, respectively. For subjects in the dominant context groups these proportions were $25.8 \%$ (test) and $0.0 \%$ (control). Actual frequencies of homograph intrusions, other intrusions, and omissions are given in Table 3.

\section{Discussion}

The results of Experiments 1 and 2 replicate those of Warren and Warren (1976) and extend their work to cover the problem of meaning dominance in the encoding of ambiguous words. Clearly, neither the unitary encoding model nor the ordered activation model can account for the pattern of intrusions observed here. Regardless of dominance or context, multiple meanings of an ambiguous word appear to be activated during encoding. Furthermore, meanings that do not match context appear to remain active in memory for a fairly long period after the selection of another meaning has taken place. Such meanings are seemingly not suppressed or deactivated as part of the selection process.

Warren and Warren (1976) have presented a multiple encoding model based on Morton's logogen system (1970) that, with appropriate modification, may account for the data obtained in the current experiments. The primary modifications needed lie in the differential representation of dominant and subordinate meanings of ambiguous words. The model suggests that verbal stimuli automatically activate stored representations in memory (logogens) sensitive to the particular combinations of physical features present in words. One such logogen is thought to exist for each word in one's vocabulary and to consist of locus for the covergence of the graphic, phonemic, and semantic features that define that word. Physical input matching the phonemic (graphic) features of a logogen leads to its activation. In addition, a logogen is activated when other words which share its semantic features are encountered (Collins \& Loftus, 1975; Warren, 1972, 1974). Activation from whatever source is combined additively and, to the degree that this activation increases, the graphic, phonemic and semantic features comprising the logogen become more salient in memory. Representations of those logogens most highly activated are selected for transfer to operational memory, and it is at this point that one presumably first becomes aware of a word.
It has been suggested (Morton, 1970) that selection of a particular logogen depends not only on the level of activation it has achieved but also on the activation exceeding a criterion level set by the frequency with which the word it represents has been encountered in the past. More frequently occurring words have logogens with lower criteria for selection and will be selected after less total activation has accumulated. In the case of homophones and homographs, it is suggested that multiple meanings are represented in memory as separate logogens with a common set of phonemic (graphic) features but with different sets of graphic (phonemic) and semantic features. Just as with other words, the criteria for these logogens will differ according to their past history of occurrence. Meaning dominance imbalance essentially reflects these differences in criteria. In fact, measures of dominance such as associative response evocation, spelling pattern, and so on are highly correlated with frequency of occurrence (Galbraith \& Taschman, 1969).

When a homophone or homograph is encountered, the multiple logogen representations of these words with their diverse semantic feature groupings must be equally activated by the physical input. The logogen whose semantic features match the context words presented concurrently must also receive activation from that source. This additional input assures that, even in the case where context matches a subordinate meaning of a word, its logogen will be the one most likely to exceed criterion first and its representation will be selected for further processing. Criterion-level differences may occasionally derail this process, however, as illustrated by the slightly different efficacy of context-trial material in biasing subordinate and dominant meanings in the current studies. Activation of unselected logogen representations-that is, activation of the semantic and physical features that define these units-must persist for some time even after selection of the other logogen has taken place.

During critical-trial presentation in the current experiments, words were presented whose semantic features matched those of the still activated logogen for an alternative meaning of the ambiguous word encountered on the context trial. The additional activation received from this source is apparently sufficient to produce the occasional selection of this meaning's logogen and the transfer of its representation to operational memory. When recall is then attempted, a scan of operational memory produces recall of the graphic or phonemic form matching this meaning, along with the actually presented material. Since they have lower criteria for selection, dominant meaning representations are more likely to be selected for transfer to operational memory than subordinate ones. The logogen activation produced by the common semantic features of criticaltrial words is apparently sufficient to insure some level of spontaneous selection of such logogens in control 
groups as well. As before, differences in criterion levels produce a greater frequency of such selection for the dominant meanings of homographs and homophones.

Though one is usually unaware of the multiple meanings of words, the current experiments illustrate that this end state of the encoding process does not reflect the dynamics of the process itself, nor the fate of residual activation at unselected meaning representations. The account provided of the present results may also allow a more precise description of instances in which one is aware of multiple meanings of a word simultaneously, as in puns. The extent to which puns succeed to aggravate (or delight) may depend on the degree to which the context they provide activates both meanings equally, relative to their respective criteria.

\section{REFERENCE NOTE}

1. Meyer, D. E., Schvaneveldt, R. W.. \& Ruddy, M. G. Activation of lexical memory. Paper presented at the meeting of the Psychonomic Society, St. Louis, Missouri, November 1972.

\section{REFERENCES}

Collins, A. M., \& Loftus, E. F. A spreading activation theory of semantic processing. Psychological Review, 1975. 82, 407-428.

Conrad, C. Context effects in sentence comprehension: A study of the subjective lexicon. Memory \& Cognition, 1974 , 2. $130-138$

Foss. D. J. Some effects of ambiguity upon sentence comprehension. Joumal of Verbal Learning and Verbal Behavior. 1970, 9, 699-706.

Foss. D. J., \& Jenkins. C. M. Some effects of context on the comprehension of ambiguous sentences. Journal of Verbal Learning and Verbal Behavior, 1973, 12, 577.589.

Galbraith, G. G.. \& Taschman, C. S. Homophone units: A normative and methodological investigation of the strength of component elements. Journal of Verbal Learning and Verbal Behavior, 1969, 8, 737-744.

Hintzman, D. L. Tables of random letters. Psychonomic Science. 1966, 5, 253-254.

Hogaboam, T. W., \& Perfetti, C. A. Lexical ambiguity and sentence comprehension. Journal of Verbal Learning and Verbal Behavior, 1975, 14, 265-274.

KUČRA, H., \& Francis, W. N. Computational analysis of present-day American English. Providence: Brown University Press, 1967.

Light, L. L., \& Carter-Sobell, L. Effects of changed semantic context on recognition memory. Joumal of Verbal Leaming and Verbal Behavior, 1970, 9, 1-11.

Morton, J. A functional model for memory. In D. A. Norman (Ed.), Models of human memory. New York: Academic Press, 1970

Myers. J. Fundamentals of experimental design. Boston: Allyn \& Bacon, 1966.

Schyaneveldt, R. W., Meyer, D. E., \& Becker, C. A Lexical ambiguity, semantic context, and visual word recognition. Journal of Experimental Psychology: Human Perception and Performance, 1976, 2, 243-256.

SWinney, D. A.. \& Hakes, D. T. Effects of prior context upon lexical access during sentence comprehension. Journal of Verbal Learning and Verbal Behavior, 1976. 15, 681-689.

WARREN, R. E. Stimulus encoding and memory. Journal of Experimental Psychology, 1972, 94, 90-100.

W ARREN, R. E. Association, directionality and stimulus encoding. Journal of Experimental Psychology, 1974. 102, 151-158.

Warren, R. E., Bresnick, J. H.. \& Green, J. P. Definitional dominance distributions for 20 English homographs. Bulletin of the Psychonomic Society. 1977, 10, 229-231.

WARREN, R. E., \& W WRren, N. T. Dual semantic encoding of homographs and homophones embedded in context. Memory \& Cognition, 1976, 4, 586-592.

WICKENS, D. D. Encoding categories of words: An empirical approach to meaning. Psychological Review', 1970, 77, 1-15.

(Received for publication October 5, 1977; revision accepted February 13, 1978.) 